\title{
THE ANALOGUE OF THE MOEBIUS GROUP OF CIRCULAR TRANSFORMATIONS IN THE KASNER PLANE*
}

\author{
JOHN DE CICCO
}

1. Introduction. We shall begin by giving some fundamental definitions. In this paper, by a curve of the plane $\pi$ we shall mean a differential element of the third order in the plane $\pi$. A simple horn-set consists of all the curves (third order differential elements) in the plane $\pi$ which possess a common point and a common direction. Let $x$ denote the curvature and $y=d x / d s$ the rate of variation of the curvature per unit length of arc $s$ of any curve of a simple horn-set at the common point. Then any curve of a simple horn-set is given by an ordered pair of numbers $(x, y)$. From this, it follows that a simple horn-set of the plane $\pi$ is a two-dimensional space, called the Kasner plane $K_{2}$, where any point of $K_{2}$ is a curve $(x, y)$ of the simple hornset. Thus to a given simple horn-set of the plane $\pi$, there is associated an auxiliary plane, called the Kasner plane $K_{2}$, such that any given point of the Kasner plane represents a curve of the simple horn-set whose curvature and rate of variation of the curvature per unit length of arc at the common point are the abscissa $x$ and the ordinate $y$ of the given point.

Kasner has shown that the group of conformal transformations in the plane $\pi$ operating on the curves of a simple horn-set induces the three-parameter group $G_{3}$ :

$$
X=m x+h, \quad Y=m^{2} y+k,
$$

where $m \neq 0, h, k$ are constants, from the points $(x, y)$ to the points $(X, Y)$ of the Kasner plane. $\dagger$ The Kasner plane is thus an affine plane.

A line consists of the $\infty^{1}$ points $C(x, y)$ of the Kasner plane which satisfy a linear equation in $x$ and $y$ with the coefficients of $x$ and $y$ not both zero. With respect to the group $G_{3}$, the lines of the Kasner plane may be classified into three distinct types.

(a) A general line is a line whose equation is of the form $y=p x+r$, where $p \neq 0$ and $r$ are constants.

(b) An infinite line is a line whose equation is of the special form $y=$ const.

* Presented to the Society, February 25, 1939.

$\dagger$ Kasner, Conformal geometry, Proceedings of the Fifth International Congress of Mathematicians, Cambridge, 1912, vol. 2. Kasner and Comenetz, Conformal geometry of horn angles, Proceedings of the National Academy of Sciences, vol. 22 (1936), pp. 303-309. 
(c) A zero line is a line whose equation is of the special form $x=$ const.

Two lines possess a simple common point or are parallel. A line parallel to neither the $x$ nor the $y$ axis is a general line, whereas a line parallel to the $x$ (or $y$ ) axis is an infinite (or zero) line.

The Kasner plane is a special type of Finsler two-space with the special Finsler metric $d s=d x^{2} / d y$. The finite form of this Finsler metric is given by (2) below. The infinite lines and the zero lines are the minimal lines of the Kasner plane. By (2), the distance between any two distinct points of an infinite (or zero) line is infinite (or zero). These statements give the reasons for the names-infinite lines and zero lines.

Under $G_{3}$, two points of the Kasner plane possess the unique invariant*

$$
\begin{gathered}
M_{12}=M\left(C_{1}, C_{2}\right)=\frac{\left(x_{2}-x_{1}\right)^{2}}{\left(y_{2}-y_{1}\right)}, \\
M_{21}=-M_{12} .
\end{gathered}
$$

Under $G_{3}$, two general lines possess the invariant $\dagger$

$$
\alpha_{12}=\alpha\left(L_{1}, L_{2}\right)=p_{2} / p_{1}, \quad \alpha_{21}=1 / \alpha_{12} .
$$

In the Kasner plane, $M_{12}$ is the distance or measure between two points and $\alpha_{12}$ is the dihorn angle between two general lines.

The set of $\infty^{1}$ points $C(x, y)$ of the Kasner plane which satisfy an equation of the form

$$
a_{0} x^{2}+a_{1} x+a_{2} y+a_{3}=0,
$$

where at least one of the coefficients of the variables is not zero, is called a parabolic-circle. With respect to the group $G_{3}$, the paraboliccircles may be classified into four distinct types.

(a) The proper parabolic-circles. Any parabolic-circle whose equation is of the form (4) where $a_{0} a_{2} \neq 0$ is called a proper parabolic-circle. This may also be defined as the set of $\infty^{1}$ points $C(x, y)$ which are at a constant distance $m$ from a fixed point $C_{0}\left(x_{0}, y_{0}\right)$. The distance $m$ is called the radius and the fixed point $C_{0}$ is called the center of the parabolic-circle. The center and radius of the proper parabolic-circle (4) are

* See the preceding papers. $M_{12}$ is the conformal measure of the horn angle formed by the two curves $C_{1}$ and $C_{2}$.

$\dagger$ Kasner, Trihornometry: a new chapter of conformal geometry, Proceedings of the National Academy of Sciences, vol. 23 (1937), pp. 337-341. 


$$
\begin{gathered}
x_{0}=-a_{1} / 2 a_{0}, \quad y_{0}=-a_{3} / a_{2}+a_{1}^{2} / 4 a_{0} a_{2}, \\
m=-a_{2} / a_{0} .
\end{gathered}
$$

For a proper parabolic-circle, the center is a finite point and the radius is a finite nonzero number.

(b) The general linear parabolic-circles. Any parabolic-circle whose equation is of the form (4) where $a_{0}=0, a_{1} \neq 0, a_{2} \neq 0$ is called a general linear parabolic-circle. It is of course a general line. We may consider this as the limiting form of a proper parabolic-circle when the center goes to infinity and the radius becomes infinite.

(c) The infinite linear parabolic-circles. Any parabolic-circle whose equation is of the form (4) where $a_{0}=0, a_{1}=0, a_{2} \neq 0$, is called an infinite linear parabolic-circle. It is an infinite line. We may consider this as the limiting form of a proper parabolic-circle when the center remains a finite point and the radius becomes infinite.

(d) The zero parabolic-circles. Any parabolic-circle whose equation is of the form (4) where $a_{2}=0$ is called a zero parabolic-circle. It consists of none (imaginary), one, or two zero lines. This may be considered as the limiting form of a proper parabolic-circle when the center remains a finite point or goes to infinity and the radius becomes zero.

From (4), it is seen that the differential equation of all paraboliccircles is $y^{\prime \prime \prime}=0$.

The problem of this paper is to obtain the group of all point transformations with nonvanishing jacobian of the Kasner plane which convert every parabolic-circle into a parabolic-circle.* Of course the Kasner group $G_{3}$ as given by equations (1) is a subgroup of this new group. It is found that this parabolic-circular group of point transformations is a continuous seven-parameter group $G_{7}$. This group $G_{7}$ is the analogue of the Moebius group of circular transformations, which is a mixed six-parameter group generated by inversions. It can be shown that our group $G_{7}$ is isomorphic to the Laguerre group of circular line transformations. This is defined as the group of all line transformations which convert every circle into a circle.

We obtain the following fundamental result. Let a point transformation $T$ of the Kasner plane convert more than $2 \infty^{2}$ purabolic-circles into parabolic-circles. That is, let T convert $3 \infty^{2}$ parabolic-circles into parabolic-circles. Then $T$ must be a parabolic-circular transformation. This gives us a minimal characterization of the parabolic-circular

* Lie has shown that the group of contact transformations which leave invariant the differential equation $y^{\prime \prime \prime}=0$ is a ten-parameter group $G_{10}$. See Lie-Scheffers, Berïhrungstransformationen, p. 241. It may be proved that if a contact transformation converts $4 \infty^{2}$ vertical parabolas into vertical parabolas, then it must be a transformation of the Lie group $G_{10}$. The same results are valid for circles. 
group $G_{7}$ of the Kasner plane.* The analogous minimal characterization for the Laguerre group is the following: Let a line transformation $T$ convert more than $2 \infty^{2}$ circles into circles. Then the line transformation $T$ must be a circular line transformation of the Laguerre group.

We close our paper with a characterization of the group of all point (or line) transformations in the plane $\pi$ by means of the paraboliccircles of the Kasner plane.

2. Parabolic-circles into parabolic-circles. Let $T$ be the point transformation with nonvanishing jacobian of the Kasner plane

$$
X=X(x, y), \quad Y=Y(x, y) .
$$

We wish to find out which parabolic-circles become parabolic-circles under $T$. In that event, the differential equation $y^{\prime \prime \prime}=0$ must be carried into $Y^{\prime \prime \prime}=0$ under the transformation $T$ as given by (6). From (6) and this last fact, we see that the parabolic-circles which become parabolic-circles under $T$ must satisfy the ordinary differential equation of the second order (of second degree in $y^{\prime \prime}$ )

where

$$
\lambda_{x}+p \lambda_{y}+p^{\prime}\left(\lambda_{p}+J_{x}+p J_{y}\right)
$$

$$
-\frac{3\left(\lambda+p^{\prime} J\right)\left(X_{x x}+2 p X_{x y}+p^{2} X_{y y}+p^{\prime} X_{y}\right)}{X_{x}+p X_{y}}=0,
$$

$$
\begin{aligned}
\lambda= & \left(X_{x}+p X_{y}\right)\left(Y_{x x}+2 p Y_{x y}+p^{2} Y_{y y}\right) \\
& -\left(Y_{x}+p Y_{y}\right)\left(X_{x x}+2 p X_{x y}+p^{2} X_{y y}\right), \\
J= & X_{x} Y_{y}-X_{y} Y_{x},
\end{aligned}
$$

and $p=y^{\prime}$. From (7) and (8), we obtain the following theorem.

THEOREM 1. Let the point transformation $T$ be such that $X_{y} \neq 0$. That is, let $T$ not convert every zero line into a zero line. Then under $T$, there are at most either (a) two distinct two-parameter families $\left(2 \infty^{2}\right)$ of parabolic-circles, or (b) one two-parameter family $\left(\infty^{2}\right)$ of paraboliccircles (in this case, the two families coincide), or (c) no parabolic-circles (in this case, the two families are imaginary), which become paraboliccircles.

* See the analogous characterizations for collineations and for the Moebius group, Kasner, The characterization of collineations, this Bulletin, vol. 9 (1903), pp. 545-546. A refined analysis of the continuity requirements of this problem is given in the Columbia dissertation of Prenowitz, Transactions of this Society, vol. 38 (1935), pp. 564-599. Also, Kasner and De Cicco, Characterization of the Moebius group of circular transformations, Proceedings of the National Academy of Sciences, vol. 25 (1939). 
Let the point transformation $T$ convert every zero line into a zero line. Then $X_{y}=0$ (hence $X_{x y}=X_{y y}=0$ ). We then find that (7) and (8) become respectively

$$
\lambda_{x}+p \lambda_{y}+p^{\prime}\left(\lambda_{p}+J_{x}+p J_{y}\right)-3\left(\lambda+p^{\prime} J\right) X_{x x} / X_{x}=0,
$$

where

$$
\lambda=X_{x}\left(Y_{x x}+2 p Y_{x y}+p^{2} Y_{y y}\right)-X_{x x}\left(Y_{x}+p Y_{y}\right), \quad J=X_{x} Y_{y} .
$$

From (9) and (10), we find that the coefficient of $p^{\prime}$ in (9) is

$$
3\left(X_{x} Y_{x y}-X_{x x} Y_{y}\right)+3 p X_{x} Y_{y y} .
$$

Before proceeding, we wish to introduce some definitions. Let two curves of the Kasner plane intersect in a given point and let their slopes at the given point be $p_{1}$ and $p_{2}$. The difference of the slopes $p_{2}-p_{1}$ is called the slope-difference between the two curves at the given point. Any point transformation of the Kasner plane which carries equal slope-differences at one point or at distinct points into equal slope-differences is termed a magnilong transformation. Obviously any such transformation is of the form

$$
X=X(x), \quad Y=\gamma X_{x} y+\beta(x),
$$

where $X$ and $\beta$ are functions of $x$ only and $\gamma$ is a constant. We observe that (12) also converts every zero line into a zero line. The set of all magnilong transformations forms a group. It can be shown that this group of transformations is isomorphic to the group of line transformations, any one of which magnifies the distance between the two points of contact on the common tangent line of any two curves. This last statement gives the reason for the name magnilong.

From (9), (10), and (11), we obtain the following result.

THEOREM 2. Let the point transformation $T$ be such that $X=X(x)$ and $Y \neq \gamma X_{x} y+\beta(x)$, where $X$ and $\beta$ are functions of $x$ only and $\gamma$ is $a$ constant. This means that $T$ is not a magnilong transformation but $T$ converts every zero line into a zero line. Then under $T$ there is at most one two-parameter family $\left(\infty^{2}\right)$ of parabolic-circles which become paraboliccircles. Of course under $T$ there are $\infty^{1}$ parabolic-circles which become parabolic-circles. These are the $\infty^{1}$ zero lines (which become zero lines).

Next let $T$ be the magnilong transformation (12). Then (9) and (10) become

$$
\lambda_{x}+p \lambda_{y}-3 \lambda X_{x x} / X_{x}=0,
$$

where 


$$
\begin{aligned}
\lambda & =\left(X_{x} \beta_{x x}-X_{x x} \beta_{x}\right)+\gamma y\left(X_{x} X_{x x x}-X_{x x}^{2}\right)+p \gamma X_{x} X_{x x}, \\
J & =\gamma X_{x}^{2}
\end{aligned}
$$

Substituting (14) into (13), we obtain

$$
\begin{aligned}
& \frac{\partial}{\partial x}\left(X_{x} \beta_{x x}-X_{x x} \beta_{x}\right)-\frac{3 X_{x x}}{X_{x}}\left(X_{x} \beta_{x x}-X_{x x} \beta_{x}\right) \\
& +\gamma y\left[\frac{\partial}{\partial x}\left(X_{x} X_{x x x}-X_{x x}^{2}\right)-\frac{3 X_{x x}}{X_{x}}\left(X_{x} X_{x x x}-X_{x x}^{2}\right)\right] \\
& \quad+\gamma p\left[2 X_{x} X_{x x x}-3 X_{x x}^{2}\right]=0 .
\end{aligned}
$$

Theorem 3. Let the point transformation $T$ be such that $X=X(x)$ $\neq(a x+b) /(c x+d)$ and $Y=\gamma X_{x} y+\beta(x)$ where $X$ and $\beta$ are functions of $x$ and $\gamma, a, b, c, d$ are constants. This means that $T$ is a magnilong transformation, but that it does not preserve the cross ratio

$$
\left(x_{3}-x_{1}\right)\left(x_{4}-x_{2}\right) /\left(x_{3}-x_{2}\right)\left(x_{4}-x_{1}\right)
$$

of every four zero lines. There are at most two one-parameter families $\left(2 \infty^{1}\right)$ of parabolic-circles which are converted into parabolic-circles. Of course, one of the two one-parameter families of parabolic-circles is the pencil of zero lines (which become zero lines).

Next let the point transformation $T$ be of the form

$$
X=\frac{a x+b}{c x+d}, \quad Y=\frac{\gamma(a d-b c)}{(c x+d)^{2}} y+\beta(x),
$$

where $\beta(x)$ is a function of $x$ only and $\gamma, a, b, c, d$ are constants. Upon substituting these values into (15), we find that the coefficient of $y$ is zero and we have

$$
\frac{\frac{\partial}{\partial x}\left(X_{x} \beta_{x x}-X_{x x} \beta_{x}\right)}{X_{x} \beta_{x x}-X_{x x} \beta_{x}}=\frac{3 X_{x x}}{X_{x}} .
$$

From (17), we obtain the following theorem.

THEOREM 4. Let the point transformation $T$ be such that

$$
X=\frac{a x+b}{c x+d}, \quad Y=\frac{e y}{(c x+d)^{2}}+\beta(x),
$$

where $\beta(x) \neq\left(f x^{2}+g x+h\right) /(c x+d)^{2}$ is a function of $x$ only and $a, b, c, d$, $e, f, g, h$ are constants. That is, $T$ is a magnilong transformation such 
that the cross ratio $\left(x_{3}-x_{1}\right)\left(x_{4}-x_{2}\right) /\left(x_{3}-x_{2}\right)\left(x_{4}-x_{1}\right)$ between every four zero lines is preserved; but it does not convert every parabolic-circle into $a$ parabolic-circle. Then the only parabolic-circles which under $T$ are converted into parabolic-circles are the $\infty^{1}$ zero lines (which of course become zero lines).

THEOREM 5. The group of point transformations $T$ of the Kasner plane which convert every parabolic-circle into a parabolic-circle is given by the equations

$$
X=\frac{a x+b}{c x+d}, \quad Y=\frac{e y+f x^{2}+g x+h}{(c x+d)^{2}},
$$

where $a, b, c, d, e, f, g, h$ are constants. Thus our group is a continuous seven-parameter group $G_{7}$.

From Theorems 1, 2, 3, 4, 5, we obtain the following conclusion.

THEOREM 6. Let a point transformation $T$ of the Kasner plane convert more than $2 \infty^{2}$ parabolic-circles into parabolic-circles. That is, let $T$ convert $3 \infty^{2}$ parabolic-circles into parabolic-circles. Then $T$ must be a parabolic-circular transformation.

THEOREM 7. The group $G_{7}$ of parabolic-circular point transformations has the following two properties:

(a) Every transformation of $G_{7}$ is a magnilong transformation. Hence every zero line is carried into a zero line.

(b) The cross ratio $\left(x_{3}-x_{1}\right)\left(x_{4}-x_{2}\right) /\left(x_{3}-x_{2}\right)\left(x_{4}-x_{1}\right)$ between every four zero lines is preserved.

We note that if in (19) we take $e$ to be the quantity $a d-b c$, the resulting six-parameter group may be written in the compact form

$$
Z=\frac{\alpha z+\beta}{\gamma z+\delta},
$$

where each of the quantities $z, Z, \alpha, \beta, \gamma, \delta$ represents a dual complex number of the form $a+j b$ where $j^{2}=0$, and $a$ and $b$ are real numbers. Of course the transformation (20) is from the point $z=x+j y$ to the point $Z=X+j Y$ of the Kasner plane.

By means of the above, we see that any transformation of the parabolic-circular group $G_{7}$ is the product of a linear fractional transformation of the form (20) followed by the transformation

$$
X=x, \quad Y=e y,
$$

where $e$ is an arbitrary real constant. 
3. The characterization of the group of all point (line) transformations in the plane $\pi$ by the parabolic-circles of the Kasner plane. It is found that the group of all contact transformations in the plane $\pi$ induces an eight-parameter group $G_{8}$ of transformations in the Kasner plane. (See Kasner, Characterization of the conformal group and the equilong group by horn angles, Duke Mathematical Journal, vol. 4 (1938), pp. 95-106.) By examining this eight-parameter group $G_{8}$, we find the following two results.

TheOREM 8. The subgroup $G_{6}$ of the parabolic-circular group $G_{7}$, which is given in the Kasner plane by the equations

$$
X=a x+b, \quad Y=e y+f x^{2}+g x+h,
$$

is induced by the group of all point transformations in the plane $\pi$ operating on the curves $C(x, y)$ (third order differential elements) of a simple horn-set.

THEOREM 9. If a contact transformation of lineal elements in the plane $\pi$ operating on the curves $C(x, y)$ (third order differential elements) of any simple horn-set converts every parabolic-circle of the Kasner plane into a parabolic-circle, then the contact transformation in the plane $\pi$ must be a point transformation.

Theorems 8 and 9 give us a new characteristic property of the group of all point transformations among all curve preserving transformations.

Let us now think of $x$ as being the radius of curvature and $y=d x / d u$ as being the rate of variation of the radius of curvature per unit radian measure of the angle $u$ that the tangent line makes with a fixed line of the curve $C$ of a simple horn-set at the common point. The Kasner group $G_{3}$ as given by the equations (1) will be induced by the group of equilong transformations. (Any transformation of this group is any line transformation such that the distance between the points of contact on the common tangent line of any two curves is preserved.) The distance $M_{12}$ will be the measure of the horn angle in equilong geometry. (See Kasner, Equilong invariants and convergence proofs, this Bulletin, vol. 23 (1917), pp. 341-347.)

Theorems 8 and 9 will be true if we substitute the word "line" for the word "point." That is, these theorems give us a characteristic property of the group of all line transformations among all curve preserving transformations.

Brooklyn College 